PHYSICAL REVIEW D 94, 029904(E) (2016)

\title{
Erratum: Nonrelativistic approximation of the Dirac equation for slow fermions coupled to the chameleon and torsion fields in the gravitational field of the Earth [Phys. Rev. D 92, 065006 (2015)]
}

A. N. Ivanov and M. Wellenzohn

(Received 15 July 2016; published 28 July 2016)

DOI: 10.1103/PhysRevD.94.029904

We add to the acknowledgements the following sentence:

This work was also supported by the Austrian "Fonds zur Förderung der Wissenschaftlichen Forschung" (FWF) under Contracts No. I862-N20 and No. P26781-N20. 